\title{
ANÁLISE DA ESTRUTURA HORIZONTAL DE UMA FLORESTA ESTACIONAL SEMIDECIDUAL LOCALIZADA NO MUNICÍPIO DE CÁSSIA-MG
}

\author{
Horizontal structural analysis of a "floresta estacional \\ semidecidual" located in the municipality of Cassia - MG
}

Márcio Coraiola *
Sylvio Péllico Netto **

Resumo

O objetivo principal do presente trabalho de pesquisa foi a caracterização estrutural de uma Floresta Estacional Semidecidual, enfocando a análise dos principais parâmetros da estrutura horizontal (abundância, dominância e freqüência). Foram utilizadas 12 unidades amostrais de 1 hectare $(100 \mathrm{~m}$ x $100 \mathrm{~m})$, distribuídas sistematicamente na área, nas quais foram medidas todas as árvores com DAP $>10 \mathrm{~cm}$. Na análise da estrutura horizontal da floresta, destacou-se o capixingui (Croton floribundus), espécie mais abundante e dominante, além do guaritá (Astronium graveolens), jambreiro (Clusiaceae 1), três folhas (Esenbeckia grandiflora), jequetibá rosa (Cariniana legalis) e ingá graúdo (Inga sp.), espécies com 100\% de freqüência absoluta na floresta.

Palavras-chave: Análise estrutural, Estrutura horizontal, Floresta natural, Manejo florestal.

\section{Abstract}

The present research paper had as main objective the structural characterization of a "Floresta Estacional Semidecidual" considering the analysis of the main horizontal structure parameters (abundance, dominance and frequency ). To conduct the study 12 sampling units with size (100m x $100 \mathrm{~m})$ were used, systematically distributed over the area, where all the trees with dbh larger than $10 \mathrm{~cm}$ were measured. In the forest horizontal structure analysis it was detected that the "capixingui" (Croton floribundus) is the most abundant and dominant species, beyond "guaritá" (Astronium graveolens), "jambreiro" (Clusiaseae 1), "três folhas" (Esenbeckia grandiflora), "jequetibá rosa" (Cariniana legalis), and "ingá graúdo" (Inga sp.) which are species with $100 \%$ absolute frequency in the forest.

Keywords: Structure analysis, Horizontal structure, Natural forest, Forest management.

* Engenheiro Florestal, M. Sc., Dr., Professor Assistente do CCAA/PUCPR.

** Engenheiro Florestal, M. Sc., Dr., Professor Titular do CCAA/PUCPR, Bolsista do CNPq. BR 376 Km 14, Costeira, São José dos Pinhais - PR, CEP 83010-500.

E-mail: pellico@rla13.pucpr.br 


\section{Introdução}

Para que haja um aproveitamento racional, aliado à sobrevivência das florestas naturais, torna-se necessário o desenvolvimento de técnicas silviculturais adequadas, baseadas na ecologia de cada tipo de formação vegetal. Conhecimentos básicos sobre a dinâmica de crescimento, recomposição florestal natural e a estrutura da floresta, propriamente dita, constituem aspectos de extrema importância para qualquer intervenção, que venha nela ser feita, sob o risco de exaurimento total de seus recursos madeireiros e dos outros que deles dependem para sobreviver. Os resultados das análises estruturais permitem fazer deduções sobre a origem, características ecológicas e sinecológicas, dinamismo e tendências do futuro desenvolvimento das florestas, elementos básicos e fundamentais para o planejamento do Manejo Silvicultural.

Enquanto não se conhece bem as características estruturais dessas florestas, não deve ocorrer seu aproveitamento racional. Por meio da análise estrutural, pode-se definir as técnicas de manejo mais adequadas para determinada região, uma vez que esta informa a composição horizontal e vertical da floresta, quantitativa e qualitativamente, permitindo, assim, definir futuras intervenções na sua estrutura, com intensidade que não comprometa sua sobrevivência futura.

Apesar da grande importância dos estudos estruturais, aliada ao seu incontestável interesse científico, poucos estudos sistemáticos a respeito têm sido realizados até o momento em florestas naturais no Brasil. Baseado nesta questão, pretende-se, com o presente trabalho, efetuar a descrição de uma Floresta Estacional Semidecidual, localizada no Município de Cássia - MG, destacando o estudo da estrutura horizontal, visando a fornecer subsídios técnicos aos profissionais da área, para o desenvolvimento de sistemas de manejo florestal eficientes.

A análise, com base em elementos estruturais quantitativos, como abundância, freqüência e dominância, foi introduzida por Cain et al. (1959), sendo crescentemente empregada em pesquisas florestais devido à sua boa aceitação (LONGHI, 1980).

Para Hosokawa (1986), as florestas naturais possuem elevada diversidade de espécies e uma grande variação de qualidades em termos econômicos. Assim, os levantamentos estruturais deverão abranger pelo menos os seguintes itens: estrutura horizontal, estrutura vertical, estrutura paramétrica e estrutura da regeneração natural.

Segundo Galvão (1994), os métodos ou procedimentos para levantamento da vegetação, de modo geral, procuram obter informações qualitativas e quantitativas da floresta, tomando como base os parâmetros da estrutura horizontal e vertical, mediante a adequação do tamanho e do número de unidades amostrais, com extensão e grau de complexidade florística da área a ser levantada.

Segundo Coraiola (1997), a floresta em questão apresentou 124 espécies, com DAP maior que 10 cm, distribuídas em 99 gêneros e 46 famílias botânicas. No caso da regeneração natural, foram encontradas apenas 69 espécies arbóreas, DAP menor que $10 \mathrm{~cm}$, distribuídas em 58 gêneros e 33 famílias botânicas. Estes resultados indicam que a diversidade de espécies da população adulta é significativamente maior que a da regeneração natural, representando quase o dobro do número de espécies.

\section{Material e métodos Área de estudo}

Os dados básicos, utilizados neste trabalho, foram obtidos em inventário florestal, realizado numa área de floresta natural, localizada no Município de Cássia, região sul do Estado de Minas Gerais, situada entre: Latitude $20^{\circ} 20^{\prime}$ e $20^{\circ} 40^{\prime}$ Sul e Longitude $46^{\circ} 40^{\prime}$ e $47^{\circ} 00^{\prime}$ Oeste. A área de estudo é formada por uma propriedade denominada como Fazenda Reata, possuindo cerca de 90 hectares de floresta natural (CORAIOLA, 1997).

A região de Cássia, MG, está situada a, aproximadamente, 680 metros de altitude e apresenta temperaturas médias anuais de $26,5^{\circ} \mathrm{C}$ (máxima) e $19,5^{\circ} \mathrm{C}$ (mínima). O clima da região, segundo classificação de Köppen, é do tipo Cwa (Tropical de altitude), apresentando verões rigorosos e chuvosos.

A vegetação predominante na região estudada é a Floresta Estacional Semidecidual. O conceito ecológico da região da Floresta Estacional está preso ao clima de duas estações, uma chuvosa e outra seca, que condicionam uma estacionalidade foliar dos elementos arbóreos dominantes, os quais têm adaptação fisiológica à deficiência hídrica ou à baixa temperatura, durante certo tempo. No caso das Florestas Semideciduais, a porcentagem de árvores caducifólias no conjunto florestal, e não das 
espécies que perdem folhas eventualmente, devese situar em torno de 20 a 50\% na época desfavorável (RADAMBRASIL, 1978).

\section{Metodologia Inventário florestal}

O inventário florestal, para realização do presente estudo, foi efetuado com base em fotografias aéreas da região e mapas da propriedade, visto que nenhum levantamento florestal prévio havia sido concretizado na área em questão. Foram instaladas 12 unidades amostrais na área, onde se considerou todos os indivíduos com DAP maior que $10 \mathrm{~cm}$.

O processo de amostragem, utilizado para o levantamento dos dados, foi o sistemático em estágio único, com intervalos constantes de 100 metros entre linhas e unidades amostrais. Com relação ao método de amostragem, foi utilizado o de área fixa, devido às inúmeras vantagens que apresenta Péllico Neto e outros (1997), bem como pela necessidade de um controle mais rígido sobre as medições e acompanhamento futuro da dinâmica da floresta (parcelas permanentes).

A unidade amostral utilizada tinha forma quadrada, com dimensões de 100 x 100 metros, resultando numa área de $10.000 \mathrm{~m}^{2}$ (1ha). Em cada árvore das parcelas foi medido o DAP, diâmetro à altura do peito, e feita a identificação das espécies pelo nome vulgar. Além das medições, cada árvore foi devidamente numerada e marcada (tinta) à altura do peito (1,30 $\mathrm{m}$ de altura do solo). Todas as árvores das unidades amostrais foram alocadas em croqui.

A identificação das espécies, usando-se o material botânico coletado, foi realizada no Laboratório de Dendrologia do Curso de Engenharia Florestal da UFPR e no Museu Botânico Municipal de Curitiba (CORAIOLA, 1997).

\section{Análise da estrutura borizontal da floresta}

A caracterização estrutural, da floresta estudada, foi realizada com base na composição florística da população e parâmetros da estrutura horizontal.

A composição florística das espécies foi analisada com base na distribuição de indivíduos, espécies, gêneros e famílias botânicas que ocorre- ram na área, para a regeneração natural e para o estrato arbóreo.

A estrutura horizontal da floresta foi analisada por meio da abundância, dominância e freqüência relativa das espécies, obtida sobre as populações adultas (indivíduos com DAP $>10 \mathrm{~cm}$ ).

Para Lamprecht (1962), a abundância mede a participação das diferentes espécies na floresta:

$$
\begin{aligned}
& A B_{\text {abs. }}=n / h a \\
& A B_{\text {rel. }}=\frac{n / h a}{N / h a} \cdot 100
\end{aligned}
$$

onde:

$A B_{a b s^{*}}=$ Abundância absoluta;

$A B_{r e l}$ = Abundância relativa;

$n / h a=$ Número de árvores de cada espécie por hectare;

$N / h a=$ Número total de árvores por hectare.

A dominância absoluta e relativa podem ser calculadas, segundo Finol (1971), pela expressão:

$$
\begin{aligned}
& D_{\text {abs. }}=g / h a \\
& D_{\text {rel. }}=\frac{g / h a}{G / h a} \times 100
\end{aligned}
$$

onde :

$D_{a b s}=$ Dominância absoluta $\left(\mathrm{m}^{2}\right)$

$D_{r e l .}=$ Dominância relativa $(\%)$

hectare

$g / h a=$ Área basal de cada espécie por

$G / h a=$ Área basal total por hectare.

Segundo Lamprecht (1964), Finol (1971)

e Vega (1968), a freqüência absoluta e relativa podem ser expressas por: uma espécie

$$
\begin{aligned}
& \begin{array}{l}
F R_{\text {rel. }}= \\
\text { sendo: }
\end{array} \frac{F R_{a b s .}}{F R_{\text {rel. }}} \times 100 \\
& F R_{\text {abs. }}=\text { Freqüência absoluta; } \\
& F R_{\text {rel. }}=\text { Freqüência relativa }(\%) .
\end{aligned}
$$




\section{Resultados e discussão} Estrutura horizontal

Os valores médios de abundância absoluta e relativa das principais espécies da floresta, em ordem decrescente de abundância absoluta, estão apresentados na TABELA 1. Como pode-se observar, as 25 espécies mais abundantes representam mais de $80 \%$ do total da floresta, destacando: Capixingui (Croton floribundus) com 10,23\%, Guaritá (Astronium graveolens) com 6,09\%, D1 (Trichillia clausennii) e Urtigão (Urera baccifera) com 6,43\%, e Marinheiro (Guarea kunthiana) com $5,93 \%$. A espécie que apresentou maior abundância em toda a floresta foi o capixingui, representando cerca de $10 \%$ do total e apresentando em média 55,25 indivíduos por hectare. Trata-se de uma espécie pioneira, de crescimento rápido, que predomina em espaços livres da floresta (clareiras).

As demais espécies, mais abundantes (guaritá, D1 e urtigão), apresentam em média 34 indivíduos por hectare, totalizando cerca de $20 \%$ do número de árvores da floresta. Com exceção do guaritá, que é uma espécie clímax e integra o estrato superior, as espécies mais abundantes não atingem grandes dimensões, ocupando os estratos inferiores da floresta.

Com relação às espécies menos abundantes, que representam cerca de $80 \%$ da composição florística da área, observa-se que agrupadas compõem cerca de $20 \%$ da abundância total da floresta. Este fato indica que, apesar da grande variedade de espécies existentes na composição florística da população, poucas espécies contribuem de maneira significativa na estrutura fitossociológica da floresta.

Silva (1990), em estudos realizados na Mata dos Godoy, Londrina-PR, destacou a presença de três espécies principais, que juntas totalizaram cerca de $75 \%$ da abundância da floresta: Trichillia clausennii, Cabralea canjerana e Guarea kunthiana. Das espécies citadas, duas (Trichillia clausennii e Guarea kunthiana) encontram-se entre as mais abundantes, também neste trabalho, comprovando, assim, a importância destas espécies para a estrutura da floresta.

TABELA 1 - abundância absoluta e relativa das principais espécies da floresta.

Table 1 - absolute and relative abundance of the most important species in the forest.

\begin{tabular}{|c|c|c|c|c|}
\hline CODIGO & NOME VULGAR & NOME CIENTIFICO & AB abs & AB rel \\
\hline 1032 & Capixingui & Croton floribundus spreng. & 55,2500 & 10,24 \\
\hline 1052 & Guarita & Astronium graveolens Jaca. & 34,8333 & 6,09 \\
\hline 1040 & D 1 & Trichillia clausennii & 34,0000 & 6,43 \\
\hline 1106 & Urtigão & Urera baccifera Gaudich. & 32,3333 & 6,44 \\
\hline 1068 & Marin heiro & Guarea kunthiana Juss. & 29,6667 & 5,94 \\
\hline 1069 & Monjoleiro & Acacia polyphyl la DC. & 27,0000 & 5,10 \\
\hline 1058 & Jambreiro & Clusiaceac 1 & 23,1667 & 3,79 \\
\hline 1045 & Farinha seca & Albizia pobycephalla (Benth) Killip. & 16,9091 & 2,97 \\
\hline 1072 & Orelha de mateiro & Chrysophyllum gonocarpum (Mart \& Eichl) Engl. & 15,3636 & 2,85 \\
\hline 1084 & Pereira & Platyciamus regnelli Benth. & 15,1000 & 2,86 \\
\hline 1096 & Quatiguá & Trichilliasp. & 14,0000 & 2,47 \\
\hline 1015 & Batal ha & Lauraceac 2 & 13,5714 & 2,25 \\
\hline 1087 & Peroba can ela de velho & A spidosperma sp. 2 & 13,2500 & 2,19 \\
\hline 1073 & Orvalho & Trichillia pallens C. & 12,4000 & 2,27 \\
\hline 1028 & Canjerana & Cabralea canjerana (Vel 1.) Martius. & 11,3333 & 2,16 \\
\hline 1082 & Pau terra & Centrolobium sp. & 10,6667 & 1,74 \\
\hline 1104 & Tres folhas & Esenbeckia grandiflora Mart. & 10,4167 & 1,87 \\
\hline 1005 & Amoreira & Maclura tinctoria (L.) Don exs teudel & 9,9091 & 1,91 \\
\hline 1042 & Embaúba & Cecropia pachystachya Treo. & 9,7778 & 1,95 \\
\hline 1035 & Caroba & Jacaranda of micrantha Cham. & 9,2500 & 1,50 \\
\hline 1062 & Jequetibá rosa & Cariniana legalis (Raddi) Kuntze & 9,2500 & 1,71 \\
\hline 1061 & Jequetibá branco & Cariniana estrellensis (Mart.) Kuntze & 9,1818 & 1,61 \\
\hline 1043 & Erva de la garto & Casearia sylvestris $\mathrm{SW}$. & 9,1429 & 1,51 \\
\hline 1022 & Cancla & Lauraceac 5 & 9,0000 & 1,78 \\
\hline 1023 & Cancla amarel a & Nectandrasp. & 9,0000 & 1,70 \\
\hline 1083 & Pau viola & Alchornea triplinervia Muell. Arg. & 8,8750 & 1,72 \\
\hline 1074 & Paineira & Chorisia speciosa St. Hill. & 8,7778 & 1,70 \\
\hline 1007 & Ingá III & Fabaccac 1 & 8,5000 & 1,35 \\
\hline 1071 & O leo de copaíba & Copaifera langsdonffii Desf. & 8,5000 & 1,41 \\
\hline 1004 & Almescla & Trichilia pallidas Sw. & 8,4545 & 1,53 \\
\hline 1006 & Ingá graúdo & Ingasp. & 8,1667 & 1,43 \\
\hline 1105 & Unha de boi & Bauhinia forficata Link. & 8,1111 & 1,52 \\
\hline 1060 & Jatobá & Hymenaea courbaril Linn. & 7,8000 & 1,24 \\
\hline 1067 & Marin heirin ho & Matayba elaeagnoides Radlk. & 7,7778 & 1,39 \\
\hline 1098 & Sangueiro & Pterocarpus violaceus Vog. C19 & 7,5455 & 1,40 \\
\hline 1041 & D 4 & Siparuna apiosyce (Mart.) DC. & 7,4545 & 1,41 \\
\hline 1076 & Palmito & Euterpe edulis Mart. & 6,7500 & 1,48 \\
\hline 1053 & G uata mbú café & A spidospema ramiflorum M. Arg. & 6,4000 & 1,34 \\
\hline 1099 & Sassafrás & Ocotea odorifera (Vel1.) Rower. & 6,3333 & 1,18 \\
\hline 1031 & Capitão & Terminalia sp. 2 & 6,1667 & 0,98 \\
\hline 1137 & Rubiaceac 2 & Rubiaceac 2 & 6,0000 & 0,99 \\
\hline 1110 & A 11 ophyllus & Allophyllus seric eus (Camb.) Radlk. & 5,6000 & 1,00 \\
\hline 1037 & Cedro & Cedrella of fissilis Vell. & 5,5556 & 1,09 \\
\hline 1090 & Peroba vermelha & Aspidospema pyric ollum Mart. & 5,5000 & 0,86 \\
\hline 1029 & Canjica & Cacsalpincaceae 1 & 5,2222 & 1,01 \\
\hline 1059 & Jaracatiá & Jaracatia spinosa (Aubl) A.DC. & 4,8000 & 0,94 \\
\hline 1054 & Imbira sapo & Lonchocarpus sp. & 4,6667 & 0,83 \\
\hline 1088 & Peroba poca & Aspidospema sp. 1 & 4,5000 & 0,72 \\
\hline 1050 & Gairova & syagrus oleraceae (Mart.) Beoc. & 4,3333 & 0,81 \\
\hline 1128 & St yrax 1 & Styrax sp. 1 & 4,3333 & 0,68 \\
\hline 1011 & Araticunzinho & Annona montana Macfad; $R$. F. Fries & 4,0000 & 0,77 \\
\hline 1055 & I pe amare lo & Tabebuia sp. 2 & 4,0000 & 0,70 \\
\hline 1077 & Panac éia & Solanum cernuum Vel1. & 4,0000 & 0,84 \\
\hline 1085 & Peroba & Aspidospema sp. 4 & 4,0000 & 0,78 \\
\hline 1092 & Pi ndaíba & Xylopia sp. & 4,0000 & 0,63 \\
\hline 1075 & Paineira branca & Pseudobombax grandiflorum (Carv.) A. Robins & 3,8333 & 0,63 \\
\hline $\begin{array}{l}1051 \\
1079\end{array}$ & Gameleiro & $\begin{array}{l}\text { Ficus sp. } 2 \\
\text { Galesia integrifolia Spreng Harms }\end{array}$ & $\begin{array}{l}3,7500 \\
3,6667\end{array}$ & 0,72 \\
\hline
\end{tabular}


A TABELA 2 apresenta a dominância absoluta e relativa média, em ordem decrescente de valores absolutos, das principais espécies encontradas na população. Observa-se que as 25 espécies dominantes representam cerca de 90\% da área basal da floresta, destacando: Capixingui (Croton floribundus) com 9,86\%, Jequetibá rosa (Cariniana legalis) com 6,39\%, Marinheiro (Guarea kunthiana) com 5,02\%, Monjoleiro (Acacia polyphylla), com 5,50\%, Sangueiro (Pterocarpus violaceus) com $5,00 \%$ e a Pereira (Platyciamus regnelli) com 4,60\%.

As 10 espécies mais abundantes representam cerca de $40 \%$ da área basal total da floresta e, considerando as 25 espécies mais abundantes, o total aumenta para 70\%. Pode-se destacar também, que seis das espécies mais abundantes da floresta aparecem também como dominantes, como é o caso do capixingui, guaritá, D1, marinheiro, monjoleiro e a pereira.
Longhi (1980), estudando uma floresta natural de Araucaria angustifolia, no Sul do Brasil, encontrou em média $23 \mathrm{~m}^{2} /$ ha de área basal, destacando que apenas sete espécies totalizaram cerca de $84 \%$ da área basal total da floresta. Silva (1990), na Mata dos Godoy, Londrina - PR, encontrou em média $40 \mathrm{~m}^{2} /$ ha de área basal, com destaque a espécie Aspidosperma polyneurum (peroba rosa), que apresentou $9,32 \mathrm{~m}^{2} /$ ha de área basal (22,8\% do total). Comparando estes resultados com os encontrados no presente trabalho, observa-se que a área basal média da floresta $\left(21,20 \mathrm{~m}^{2} / \mathrm{ha}\right)$ está baixa, principalmente quando comparada com a Mata dos Godoy (Floresta Estacional Semidecidual). Este fato pode ser explicado pela grande quantidade de árvores de pequenas dimensões (DAP), e pela baixa abundância das árvores de grande porte na floresta (árvores isoladas).

TABELA 2 - dominância absoluta e relativa das principais espécies da floresta

Table 2 - absolute and relative dominance of the most important species in the forest

\begin{tabular}{|c|c|c|c|c|}
\hline CÓDIGO & $\begin{array}{l}\text { NOME } \\
\text { VULGAR }\end{array}$ & NOME CIENTIFICO & D abs & D rel \\
\hline 1032 & Capixingui & Croton floribundus Spreng. & 1,98 & 9,83 \\
\hline 1062 & Jequetibá rosa & Cariniana legalis (Raddi) Kuntze & 1,44 & 6,40 \\
\hline 1068 & Marinheiro & Guarea kunthiana Juss. & 1,26 & 5,02 \\
\hline 1098 & Sangu eiro & Pterocarpus violaceus Vog. $\mathrm{C} 19$ & 1,16 & 5,07 \\
\hline 1069 & Monjol eiro & Acacia polyphylla DC. & 1,05 & 5,59 \\
\hline 1084 & Pereira & Platyciamus regnelli Benth. & 1,03 & 4,62 \\
\hline 1052 & Guaritá & Astronium graveolens Jacq. & 0,92 & 4,59 \\
\hline 1090 & Peroba vermelha & Aspidosperma pyricollum Mart. & 0,86 & 4,21 \\
\hline 1039 & $\mathrm{D}$ & Desconhecida & 0,80 & 3,92 \\
\hline 1074 & Paineira & Chorisia speciosa St. Hill. & 0,79 & 3,94 \\
\hline 1040 & D1 & Trichillia clausennii & 0,73 & 3,02 \\
\hline 1005 & Amoreira & Maclura tinctoria (L.) Don ex Ste udel & 0,72 & 3,25 \\
\hline 1046 & Figueira & Ficus sp. 1 & 0,72 & 3,01 \\
\hline 1106 & Urtigão & Urera baccifera Gaudich. & 0,69 & 2,99 \\
\hline 1061 & Jequetibá branco & Cariniana estrellensis (Mart.) Kuntze & 0,67 & 3,23 \\
\hline 1071 & Óleo de copaíba & Copaifera langsdorffii Desf. & 0,65 & 3,98 \\
\hline 1083 & Pau viola & Alchornea triplin ervia Muell. Arg. & 0,62 & 2,50 \\
\hline 1001 & Açoita cavalo & Cordia sp. 2 & 0,56 & 2,64 \\
\hline 1007 & Ingá III & Fabac eae 1 & 0,55 & 2,69 \\
\hline 1102 & Tamborilo & Enterolobium contorstisiliquum Morong. & 0,53 & 2,21 \\
\hline 1015 & Batalha & Lauraceae 2 & 0,51 & 2,45 \\
\hline 1016 & Bico de pato & Machaerium aculeatum Raddi & 0,50 & 2,27 \\
\hline 1054 & Imbira sapo & Lonchocarpus sp. & 0,48 & 2,03 \\
\hline 1051 & Gameleiro & Ficus sp. 2 & 0,46 & 1,79 \\
\hline
\end{tabular}


TABELA 2 - dominância absoluta e relativa das principais espécies da floresta (cont.)

Table 2 - absolute and relative dominance of the most important species in the forest (cont.)

\begin{tabular}{|c|c|c|c|c|}
\hline CÓDIGO & $\begin{array}{l}\text { NOME } \\
\text { VULGAR }\end{array}$ & NOME CIENTÍFICO & D abs & D rel \\
\hline 1075 & Paineira branca & Pseudobombax grandiflorum (Carv.) A. Robins & 0,45 & 2,06 \\
\hline 1060 & Jatobá & Hymenaea courbaril Linn. & 0,45 & 2,56 \\
\hline 1109 & Vinhático & Vochysia tucan orum Mart. & 0,43 & 1,84 \\
\hline 1058 & Jambreiro & Clusiaceae 1 & 0,41 & 2,37 \\
\hline 1037 & Cedro & Cedrella cf. fissilis Vell. & 0,41 & 1,68 \\
\hline 1072 & Orelha de mateiro & Chrysophyllum gonoca rpum (Mart \& Eichl) Engl. & 0,41 & 1,88 \\
\hline 1079 & Pau alho & Galesia integrifolia S preng. Harms. & 0,41 & 1,88 \\
\hline 1028 & Canjerana & Cabralea canjerana (Ve11.) Martius. & 0,39 & 1,69 \\
\hline 1059 & Jaracatiá & Jaracatia spinosa (Aubl) A.DC. & 0,39 & 1,84 \\
\hline 1021 & Canafístula & Cassia ferruginea Schrad. ex DC. & 0,36 & 1,66 \\
\hline 1053 & Guatambú café & Aspidosperma ramiflorum M. Arg. & 0,32 & 1,37 \\
\hline 1022 & Canela & Lauraceae 5 & 0,31 & 1,32 \\
\hline 1045 & Farinha seca & Albizia polycephalla (B enth) Killip. & 0,31 & 1,81 \\
\hline 1042 & Embaúba & Cecropia pachystachya Trec. & 0,30 & 1,25 \\
\hline 1023 & Canela amarela & Nectandra sp. & 0,29 & 1,16 \\
\hline 1085 & Peroba & Aspidosperma sp. 4 & 0,28 & 1,28 \\
\hline 1087 & Peroba canela de velho & Aspidosperma sp. 2 & 0,26 & 1,28 \\
\hline 1073 & Orvalh o & Trichillia pallens $\mathrm{C}$. & 0,25 & 1,14 \\
\hline 1029 & Canjica & Caesalpineaceae 1 & 0,24 & 1,00 \\
\hline 1067 & Marinheirinho & Matayba elaeagnoides Radlk. & 0,23 & 1,17 \\
\hline 1096 & Quatiguá & Trichillia sp. & 0,21 & 0,95 \\
\hline 1035 & Caroba & Jacaranda cf. micrantha Cham. & 0,20 & 1,07 \\
\hline 1082 & Pau terra & Centrolobium sp. & 0,20 & 1,21 \\
\hline 1089 & Peroba rosa & Aspidosperma polyneu ron Muell. Arg. & 0,19 & 0,92 \\
\hline 1002 & Alecrim & Holocalix balansae Micheli & 0,19 & 0,71 \\
\hline 1115 & Sobraji & Columbrina glandulosa Perkins & 0,19 & 0,82 \\
\hline 1099 & Sassafrás & Ocotea pretiosa Benth. \& Hook. & 0,19 & 0,89 \\
\hline 1031 & Capitão & Terminalia sp. 2 & 0,18 & 0,88 \\
\hline 1095 & Quares ma & Miconia dis color DC. & 0,16 & 0,77 \\
\hline 1041 & D4 & Siparuna apiosyce (Mart.) DC. & 0,16 & 0,66 \\
\hline 1043 & Erva de lagarto & Casearia sylvestris $\mathrm{Sw}$. & 0,15 & 0,88 \\
\hline 1006 & Ingá graúdo & Inga sp. & 0,15 & 0,80 \\
\hline 1010 & Aratic um cagão & Annona cacans Warms. & 0,13 & 0,65 \\
\hline 1105 & Unha de boi & Ba uhiniaforficata Link. & 0,13 & 0,63 \\
\hline
\end{tabular}

As freqüências, absoluta e relativa, das principais espécies integrantes da área estudada são apresentadas na TABELA 3, organizada em ordem decrescente de valores absolutos. Observase que somente as espécies capixingui (Croton floribundus), guaritá (Astronium graveolens), jambreiro (Clusiaceae 1), três folhas (Esenbeckia grandiflora), jequetibá rosa (Cariniana legalis) e ingá graúdo (Inga sp.) ocorrem em todas as parcelas levantadas, apresentando uma distribuição uniforme em toda a floresta.

Observa-se, também, que das 124 espécies encontradas na floresta, 57 ocorrem em pelo menos 50\% das parcelas, ou seja, apresentam pelo menos 50\% de freqüência absoluta. Pode-se destacar, também, que das 6 espécies mais freqüentes na população, 2 encontram-se entre as mais abun- dantes e dominantes da floresta, que é o caso do capixingui e do guaritá, indicando, desta forma, a importância fitossociológica destas espécies para a estrutura da floresta. Destaca-se, também, as espécies jambreiro e três folhas, com ampla distribuição na floresta (alta freqüência e alta abundância), representadas por indivíduos de pequenas dimensões (baixa dominância). No caso do jequetibá rosa (100\% de freqüência absoluta), observou-se que, mesmo não apresentando uma abundância absoluta alta, possui a segunda maior dominância absoluta da floresta, indicando a presença de indivíduos de grande porte uniformemente distribuídos na floresta.

Silveira (1993), em estudos realizados na Floresta Estacional Semidecidual da região de Londrina-PR, Mata dos Godoy, observou que nenhu- 
ma das espécies amostradas obteve 100\% de freqüência absoluta, destacando: a Nectandra megapotamica, com $70 \%$ de freqüência absoluta e $5,38 \%$ de freqüência relativa, e a Cabralea canjerana, com $63,33 \%$ e 4,38\% respectivamente para as freqüências absoluta e relativa. Silva (1990), em trabalho desenvolvido na mesma região, obteve resultados semelhantes, onde nenhuma espécie apresentou 100\% de freqüência absoluta, destacando: Aspidosperma polyneurum, com 96\%,
Trichillia clausennii e Cabralea canjerana com $88 \%$. Com relação à floresta estudada, observouse que seis espécies apresentaram 100\% de freqüência absoluta, comprovando a importância das mesmas na estrutura da floresta. Das espécies mais freqüentes, citadas por Silva (1990) e Silveira (1993), todas ocorreram também na área estudada, neste trabalho, demonstrando a ampla distribuição destas espécies no Bioma: Floresta Estacional Semidecidual.

TABELA 3 - freqüência absoluta e relativa das principais espécies da floresta

Table 3 - absolute and relative frequence of the most important species in the forest

\begin{tabular}{|c|c|c|c|c|}
\hline CÓDIGO & $\begin{array}{l}\text { NOME } \\
\text { VULGAR }\end{array}$ & NOME CIENTIFICO & F abs & Frel \\
\hline 1006 & Ingá graúdo & Inga sp. & 100,000 & 1,81 \\
\hline 1032 & Capixingui & Croton floribundus Spreng. & 100,000 & 1,81 \\
\hline 1039 & $\mathrm{D}$ & Desc onhecida & 100,000 & 1,81 \\
\hline 1052 & Guaritá & Astronium graveolens Jacq. & 100,000 & 1,81 \\
\hline 1058 & Jambreiro & Clusiaceae 1 & 100,000 & 1,81 \\
\hline 1062 & Jequetibá rosa & Cariniana legalis (Radd i) Kuntze & 100,000 & 1,81 \\
\hline 1104 & Três folhas & Esenbeckia grandiflora Mart. & 100,000 & 1,81 \\
\hline 1004 & Almescla & Trichilia pallida $\mathrm{Sw}$. & 91,667 & 1,66 \\
\hline 1005 & Amoreira & Maclura tinctoria (L.) Don ex Steudel & 91,667 & 1,66 \\
\hline 1041 & D4 & Siparuna apiosyce (Mart.) DC. & 91,667 & 1,66 \\
\hline 1045 & Farinha seca & Albizia polycephalla (Benth) Killip. & 91,667 & 1,66 \\
\hline 1061 & Jequetibá branco & Cariniana estrellensis (Mart.) Kunte & 91,667 & 1,66 \\
\hline 1072 & Orelha de mateiro & Chrysophyllum gonocarpum (Mart \& Eichl) Engl. & 91,667 & 1,66 \\
\hline 1096 & Quatiguá & Trichillia sp. & 91,667 & 1,66 \\
\hline 1098 & Sangu eiro & Pterocarpus violaceus Vog. C19 & 91,667 & 1,66 \\
\hline 1019 & Camboatá & Cupania vernalis Camb. & 83,333 & 1,51 \\
\hline 1022 & Canela & Lauraceae 5 & 83,333 & 1,51 \\
\hline 1040 & D1 & Trichillia clausennii & 83,333 & 1,51 \\
\hline 1059 & Jaracatiá & Jaracatia spinosa (Aubl) A.DC. & 83,333 & 1,51 \\
\hline 1073 & Orvalh o & Trichillia pallens $\mathrm{C}$. & 83,333 & 1,51 \\
\hline 1084 & Pereira & Platyciamus regnelli Benth. & 83,333 & 1,51 \\
\hline 1010 & Araticum cagão & Annona cacans Warms. & 75,000 & 1,36 \\
\hline 1023 & Canela amarela & Nectandra sp. & 75,000 & 1,36 \\
\hline 1028 & Canjerana & Cabralea canjerana (Vell.) Martius. & 75,000 & 1,36 \\
\hline 1029 & Canjica & Caesalpineaceae 1 & 75,000 & 1,36 \\
\hline 1033 & Capororocão & Myrsine umbell ata Mez. & 75,000 & 1,36 \\
\hline 1037 & Cedro & Cedrella cf. fissilis Vell. & 75,000 & 1,36 \\
\hline 1042 & Embaúba & Cecropia pachystachya Trec. & 75,000 & 1,36 \\
\hline 1067 & Marinheirinho & Matayba elaeagnoides Radlk. & 75,000 & 1,36 \\
\hline 1068 & Marinheiro & Guarea Kunthian a Juss. & 75,000 & 1,36 \\
\hline 1069 & Monjoleiro & Acacia polyphylla DC. & 75,000 & 1,36 \\
\hline 1074 & Paineira & Chorisia speciosa St. Hill. & 75,000 & 1,36 \\
\hline 1099 & Sassafrás & Ocotea odorifera (Vell.) Rohwer. & 75,000 & 1,36 \\
\hline 1105 & Unha de boi & Bauhinia forficata Link. & 75,000 & 1,36 \\
\hline 1106 & Urtigão & Urera baccifera Gaudich. & 75,000 & 1,36 \\
\hline
\end{tabular}


TABELA 3 - freqüência absoluta e relativa das principais espécies da floresta (cont.)

Table 3 - absolute and relative frequence of the most important species in the forest (cont.)

\begin{tabular}{|c|c|c|c|c|}
\hline \multicolumn{2}{|c|}{$\begin{array}{ll}\text { CÓDIGO } & \text { NOME } \\
& \text { VULGAR }\end{array}$} & \multirow{2}{*}{$\begin{array}{l}\text { NOME CIENTÍFICO } \\
\text { Cassia ferruginea } \text { Schrad. ex DC. }\end{array}$} & \multirow{2}{*}{$\begin{array}{l}\text { F abs } \\
66,667\end{array}$} & \multirow{2}{*}{$\begin{array}{c}\text { Frel } \\
1,21\end{array}$} \\
\hline & & & & \\
\hline 1051 & G amele iro & Ficus sp. 2 & 66,667 & 1,21 \\
\hline 1083 & Pau viola & Alchornea triplin ervia Muell. Arg. & 66,667 & 1,21 \\
\hline 1089 & Peroba rosa & Aspidosperma polyneuron Muell. Arg. & 66,667 & 1,21 \\
\hline 1091 & Pessegueiro b ravo & Prunus subcoriacea Koehne. & 66,667 & 1,21 \\
\hline 1015 & Batalha & Lauraceae 2 & 58,333 & 1,06 \\
\hline 1043 & Erva de lagarto & Casearia sylvestris $\mathrm{Sw}$. & 58,333 & 1,06 \\
\hline 1101 & Sete casaco & Myrtaceae 1 & 58,333 & 1,06 \\
\hline 1115 & Sobraji & Columbrina glandulosa Perkins & 58,333 & 1,06 \\
\hline 1002 & A lecrim & Holocalix balansae Micheli & 50,000 & 0,90 \\
\hline 1008 & Ingá miúdo & Inga marginata Wild. & 50,000 & 0,90 \\
\hline 1011 & Araticunzinho & Annona montana Macfad; R. F. Fries & 50,000 & 0,90 \\
\hline 1016 & Bico de pato & Machaerium aculeatum Raddi & 50,000 & 0,90 \\
\hline 1031 & Capitão & Terminalia sp. 2 & 50,000 & 0,90 \\
\hline 1034 & Carne de vaca & Roupala sp. & 50,000 & 0,90 \\
\hline 1046 & Figueira & Ficus sp. 1 & 50,000 & 0,90 \\
\hline 1050 & G airova & Syagrus oleraceae (Mart.) Becc. & 50,000 & 0,90 \\
\hline 1054 & Imbira sapo & Lonchocarpus sp. & 50,000 & 0,90 \\
\hline 1055 & I pê amarelo & Tabebuia sp. 2 & 50,000 & 0,90 \\
\hline 1065 & Mamica de porca & Zanthoxylum sp. & 50,000 & 0,90 \\
\hline 1075 & Pa ineira branca & Pseudobombax grandiflorum (Carv.) A. Robins & 50,000 & 0,90 \\
\hline 1080 & Pau ferro & Myrtaceae 3 & 50,000 & 0,90 \\
\hline 1017 & Braúna & Schinopsis brasili ensis Engl. & 41,667 & 0,75 \\
\hline 1025 & Canela branca & Lauraceae 4 & 41,667 & 0,75 \\
\hline 1053 & Guatambú café & Aspidosperma ramiflorum $\mathrm{M}$. Arg. & 41,667 & 0,75 \\
\hline 1060 & Jatobá & Hymenaea courbaril Linn. & 41,667 & 0,75 \\
\hline 1092 & Pindaíba & Xylopia sp. & 41,667 & 0,75 \\
\hline 1100 & Serralha & Sorocea guillerminiania Gaudich. & 41,667 & 0,75 \\
\hline 1110 & A llophyllus & Allophyllus sericeus (Camb.) Radlk. & 41,667 & 0,75 \\
\hline 1003 & Amendoim & Senna sp. 1 & 33,333 & 0,60 \\
\hline 1018 & Cambará lixa & Aloysia virginata Juss. & 33,333 & 0,60 \\
\hline 1035 & Caroba & Jacaranda cf. micrantha Cham. & 33,333 & 0,60 \\
\hline 1076 & Pa lmito & Euterpe edulis Mart. & 33,333 & 0,60 \\
\hline 1085 & Peroba & Aspidosperma sp. 4 & 33,333 & 0,60 \\
\hline 1086 & Peroba branca & Aspidosperma sp. 3 & 33,333 & 0,60 \\
\hline 1087 & Peroba can ela de velho & Aspidosperma sp. 2 & 33,333 & 0,60 \\
\hline 1103 & T ento & Ormosia arborea Harnu. & 33,333 & 0,60 \\
\hline 1013 & Arruda & Zanthoxylum rhoifolium Lam. & 25,000 & 0,45 \\
\hline
\end{tabular}

\section{Conclusões}

$\mathrm{Na}$ estrutura horizontal da floresta, destaca-se o capixingui (Croton floribundus), a espécie mais importante da floresta, apresentando os maiores valores relativos à abundância, dominância e freqüência. As espécies mais abundantes da floresta, que representam cerca de $35 \%$ do total, são: capixingui (Croton floribundus), guaritá (Astronium graveolens), D1 (Trichillia clausennii), urtigão (Urera baccifera) e marinheiro (Guarea kunthiana). Com relação à dominância, as espécies que apresentaram os maiores valores foram o capixingui (Croton floribundus), jequetibá rosa (Cariniana legalis), marinheiro (Guarea kunthiana), monjoleiro (Acacia polyphylla) e sangueiro (Pterocarpus violaceous), contribuindo com aproximadamente $32 \%$ da área basal total da floresta. Entre as espécies encontradas, apenas 6 delas, capixingui (Croton floribundus), guaritá (Astronium graveolens), jambreiro (Clusiaseae 1), três folhas (Esenbeckia grandiflora), jequetibá rosa (Cariniana legalis) e ingá graúdo (Inga sp.), são comuns em toda a área da floresta (100\% de freqüência), sendo, portanto, as espécies que mais caracterizam a associação florestal em questão. 


\section{Agradecimentos}

A todos que contribuíram, direta ou indiretamente, para a realização deste trabalho, especialmente: aos Engenheiros Florestais e amigos, Alexandre Koehler, Dennis Dosza, Fernando José Fabrowski e Sandro Dallacorte, pelos trabalhos de campo e manipulação dos dados; aos professores Carlos Roberto Sanquetta, Carlos Vellozo Roderjan e Willian Thomaz Wendling, pela contribuição e auxílio; aos participantes dos trabalhos de campo, Sr. Antônio Delfino Ferreira e Eduardo Ferreira; ao Curso de Pós-Graduação em Engenharia Florestal da Universidade Federal do Paraná, pela oportunidade concedida; a Cris e a minha família, pelo auxílio nos momentos difíceis.

\section{Referências}

CAIN, S. A.; CASTRO, G. M. de Oliveira. Manual of vegetation analysis. New York: Hafner Publishing Company, 1959. 325 p.

CORAIOLA, M. Caracterização estrutural de uma Floresta Estacional Semidecidual localizada no Município de Cássia - MG, 1997. 196 f. Dissertação (Mestrado) - UFPR. Curitiba, 1997.

FINOL U., H. Nuevos parametros a considerarse en el analisis estructural de las selvas vigentes tropicales.

Rev. For. Venez., v. 14, n.21, p. 29-42, 1971.

GALVÃO, F. Métodos de levantamento fitossociológico: a vegetação natural do Estado do Paraná. Curitiba: IPARDES, 1994.

LAMPRECHT, H. Ensayo sobre unos metodos para el Análisis Estructural de los bosques tropicales. Acta Cientifica Venezolana, v. 13, n. 2, p. 57-65, 1962.

LONGHI, S. J. A estrutura de uma floresta natural de Araucaria angustifolia (Bert.) O. Ktze, sul do Brasil. Curitiba, 1980. 198 f. Dissertação (Mestrado) - UFPR, 1980.

PÉLLICO NETTO, S.; BRENA, D. A. Inventário Florestal. Curitiba, 1997. v.1, 195 p

RADAMBRASIL. Levantamento de recursos naturais, [S. 1.: s. n.], 1978. v. 32.

SILVA, S. L. H. Fitossociologia arbórea da porção norte do Parque Estadual Mata dos Godoy, Londrina - PR. Curitiba, 1990. 197 f. Dissertação (Mestrado) - UFPR, 1990.

SILVEIRA, Marcos. Estudo vegetacional em uma toposseqüência no Parque Estadual “ Mata dos Godoy “, Londrina - PR. Curitiba, 1993. 142 f. Dissertação (Mestrado).- UFPR, 1993.

VEGA, C. L. La estructura y composicion de los bosques humedos tropicales del Carare. Colombia. Turrialba, v. 18 n. 4, p. 416-436, 1968. 